\title{
El nuevo Reglamento del Tribunal de Justicia de la Unión Europea*
}

\author{
Isaac Soca Torres \\ Departamento de Derecho Privado \\ Facultad de Derecho (ESADE), Universidad Ramon Llull
}

\begin{abstract}
Sumario: I. Razones del cambio y objetivos del nuevo Reglamento.-II. La organización del Tribunal.-III. Las disposiciones de procedimiento comunes. - IV. Las cuestiones prejudiciales. - V. Los recursos directos. - VI. Los recursos de casación contra las resoluciones del Tribunal General.-VII. El reexamen de las resoluciones del Tribunal General. - VIII. Los dictámenes. - IX. Los procedimientos especiales.$\mathrm{X}$. Consideraciones finales.
\end{abstract}

Resumen: Frente al constante incremento del número de asuntos que le son sometidos, con un claro predominio de las cuestiones prejudiciales, el Tribunal de Justicia de la Unión Europea ha modificado sus reglas de procedimiento para adaptarse mejor a las especificidades de estos asuntos. El artículo comienza explicando las razones concretas que han llevado el Tribunal a cambiar y a refundir sus normas de procedimiento. A continuación, analiza los principales cambios y novedades. Para facilitar la consulta, la estructura del análisis sigue la nueva estructura del Reglamento. El artículo concluye, en resumen, que en relación a la creciente carga de asuntos, el nuevo Reglamento podría no tener un impacto material, si no se realizan cambios estructurales en el Tribunal.

Palabras clave: UE, TJUE, Reglamento de Procedimiento, instituciones, justicia.

Abstract: Faced with a constant rise in the number of cases brought before it, dominated by references for a preliminary ruling, the Court of Justice of the European Union has adapted its rules of procedure to ensure that the particular features of those cases can more readily be taken into consideration. The article starts explaining the specific reasons which led the Court to change and recast its rules of procedure. Then, it analyses the main changes and novelties. For easy reference, the structure of analysis follows the new structure of the rules. The article concludes, in short, that as regards with the growing caseload, the new rules might not have a material impact without making structural changes in the Court.

Keywords: EU, ECJ, Rules of Procedure, institutions, justice.

* Recibido el 14 de enero de 2013, aceptado el 12 de junio 2013. 


\section{Razones del cambio y objetivos del nuevo Reglamento}

El Diario Oficial de la Unión Europea publicó el 29 de septiembre de 2012 la nueva versión del Reglamento de Procedimiento del Tribunal de Justicia de la Unión Europea, que fue adoptado el 25 de septiembre del mismo año, y cuya entrada en vigor se pospuso hasta el día 1 de noviembre de $2012^{1}$.

El Tribunal de Justicia, como las demás instituciones de la Unión Europea, disponía desde su origen de un Reglamento destinado a establecer sus normas esenciales de organización y funcionamiento y a precisar detalladamente el modo en que se desarrolla el procedimiento seguido ante él. No obstante, el mencionado Reglamento, si bien a lo largo de los años había sufrido sucesivas modificaciones ${ }^{2}$, en su estructura fundamental no había cambiado desde la fecha de aprobación inicial, el 4 de marzo de $1953^{3}$. Así el Reglamento de Procedimiento seguía reflejando la preponderancia de los recursos directos, en los que, habitualmente, una persona física o jurídica o un Estado miembro se enfrenta a una institución de la Unión, mientras que, de hecho, este tipo de asuntos ya no son en su mayoría competencia del Tribunal de Justicia, con excepción de los recursos por incumplimiento y de ciertos tipos específicos de recursos de anulación ${ }^{4}$. Al finalizar el año 2011, por ejemplo, de entre los asuntos sometidos al Tribunal de Justicia, el grupo más numeroso lo constituyeron las cuestiones prejudiciales presentadas por juzgados y tribunales de los Estados miembros, que alcanzaron el sesenta por ciento 5 .

${ }^{1}$ RPTJ (Reglamento de Procedimiento del Tribunal de Justicia), DO L 265 de 29 de septiembre de 2012. La publicación y la entrada en vigor del Reglamento se encuentran previstas en su artículo 210.

${ }^{2} \mathrm{Al}$ respecto de las referidas modificaciones es muy ilustrativa la relación comentada que ofrece NAÔMÉ, C., Le renvoi préjudiciel en droit européen. Guide pratique, Bruselas, 2010, pp. 15-37.

3 Journal officiel de la Communauté européenne del charbon et de l'acier, de 7 de marzo de 1953 , p. 37.

${ }^{4}$ RPTJd (Reglamento de Procedimiento del Tribunal de Justicia derogado) DO L 176 de 4 de julio de 1991, en su versión modificada en último lugar el 23 de marzo de 2010 (DO L 92 de 13 abril de 2010).

5 Así, en el año 2011, se sometieron al Tribunal de Justicia 688 asuntos nuevos (con independencia de las acumulaciones por razón de conexión), lo que representa un aumento significativo respecto del año 2010 (631 asuntos interpuestos) y, por segundo año consecutivo, el número más elevado en la historia de la institución. Lo mismo ocurre en relación con las peticiones de decisión prejudicial. El número de asuntos prejudiciales interpuestos en el año 2011, por tercer año consecutivo, el más alto jamás alcanzado y, en comparación con el año 2009, registra un aumento de casi un $41 \%$ (423 asuntos en 2011 frente a 302 asuntos en 2009). Asimismo, cabe señalar el notable aumento del número de recursos de casación (162 en 2011 frente a 97 en 2010) y la disminución del número de recursos directos, y esto, por 
A la vista de esta realidad, marcada por el constante incremento del número de asuntos que le son sometidos, con un claro predominio de las cuestiones prejudiciales, el Tribunal de Justicia, presentó al legislador de la Unión una propuesta de refundición y actualización de su Reglamento de Procedimiento, dirigida principalmente a mejorar la eficacia de los procedimientos ante los órganos jurisdiccionales de la Unión ${ }^{6}$.

La referida propuesta del Tribunal de Justicia se encauzó a partir de cuatro objetivos concretos:

Primero. Reservar un título específico para las cuestiones prejudiciales y, al mismo tiempo completar y hacer más explícitas las reglas contenidas en él, tanto para los justiciables como para los tribunales nacionales.

Segundo. Proseguir con los esfuerzos del Tribunal de Justicia, para conseguir que el mismo pudiera mantener su capacidad de resolver dentro de un plazo razonable los asuntos que le son sometidos, cuyo número va progresivamente en aumento. Tal voluntad correspondía, por lo demás, a una exigencia inscrita en el artículo 47, párrafo segundo, de la Carta de los Derechos Fundamentales de la Unión Europea, que adquirió el mismo valor jurídico que los Tratados desde la entrada en vigor del Tratado de Lisboa, el 1 de diciembre de $2009^{7}$.

Guiado por el afán de reducir la duración de los procedimientos desarrollados ante él, particularmente en el caso de los asuntos prejudiciales, en los que el procedimiento ante el tribunal remitente queda suspendido a la espera de la decisión del Tribunal de Justicia, este último emprendió ya a partir de 2003 una reflexión sobre sus métodos de trabajo y adoptó una serie de medidas, tanto a nivel práctico como legislativo, destinadas a alcanzar este objetivo. Entre estas medidas pueden citarse, por ejemplo, la posibilidad de que las partes pudieran presentar escritos procesales ante el Tribunal de Justicia por fax u otros medios técnicos de comunicación, la reducción de los plazos de intervención como coadyuvante en el procedi-

quinto año consecutivo. Los recursos directos solo representan ya alrededor del $12 \%$ de los asuntos interpuestos, mientras que suponían aproximadamente un $38 \%$ en 2007 (la actividad y estadísticas del Tribunal de Justicia se encuentran disponibles en su página web http://curia. europa.eu).

${ }^{6}$ El proyecto del nuevo RPTJ se sometió a aprobación del Consejo de la Unión Europea el 30 de mayo de 2011, de conformidad con el artículo 253, párrafo sexto, del TFUE, disposición igualmente aplicable al TCEEA en virtud del artículo 106 bis de este último Tratado (el referido documento puede encontrarse en el registro público del Consejo de la Unión Europea http://register.consilium.europa.eu).

${ }^{7}$ Es preciso subrayar que, a pesar de este incremento, el Tribunal de Justicia ha conseguido reducir significativamente el periodo medio de tramitación de los asuntos. Así, por ejemplo, en 2011 el periodo medio de tramitación de las cuestiones prejudiciales fue de 16,4 meses, mientras que en 2003 era de 25 meses (la actividad y estadísticas del Tribunal de Justicia se encuentran disponibles en su página web http://curia.europa.eu). 
miento en los recursos directos y en los recursos de casación, la posibilidad de tramitar tales recursos - o las peticiones de decisión prejudicial- mediante un procedimiento acelerado o, por último, la creación de un procedimiento específico para los asuntos relacionados con el espacio de libertad, seguridad y justicia: el procedimiento prejudicial de urgencia, que entró en vigor el 1 de marzo de 2008.

El referido proyecto de Reglamento era continuación directa de tal reflexión y también pretendía incorporar normas que permitieran mejorar aún más la eficacia del trabajo del Tribunal de Justicia, mediante un uso óptimo de los recursos disponibles. Entre las modificaciones propuestas en este sentido pueden mencionarse la posibilidad de enviar y recibir escritos procesales por vía electrónica, la posibilidad ofrecida a los Estados miembros y a las instituciones de la Unión mencionados en el artículo 40, párrafo primero, del Estatuto de intervenir como coadyuvantes en los litigios sometidos al Tribunal de Justicia mediante una simple declaración, la simplificación de los procedimientos previos a la adopción de un auto en los supuestos en que la cuestión planteada por un tribunal nacional no suscitara ninguna duda razonable o, por último, la facultad del Tribunal de Justicia de resolver sin celebrar una vista al considerar que el conjunto de observaciones escritas presentadas ante él ofrecía información suficiente.

Tercero. Además de querer optimizar la eficacia de su trabajo, el Tribunal de Justicia se mostraba en el proyecto firmemente decidido a clarificar las normas procesales. En efecto, con objeto de permitir que todos los participantes en el procedimiento pudieran discernir con precisión sus derechos y sus obligaciones, el Tribunal estimaba oportuno codificar algunas de las normas o prácticas existentes, tales como la atribución del examen de la solicitud de asistencia jurídica gratuita a la Sala de tres Jueces a la que estuviera adscrito el Juez encargado del asunto en el que se hubiera presentado la solicitud, o la posibilidad de que el Tribunal organizara vistas comunes a varios asuntos o proteja el anonimato de ciertas personas.

Siguiendo la misma línea, de búsqueda de la efectividad, se eliminaron del proyecto de Reglamento ciertas normas inaplicadas o en desuso, al tiempo que se acortaron o simplificaron otras normas excesivamente detalladas. Así fue, en particular, con las disposiciones relativas a la Secretaría y a los testigos o peritos, y sobre todo con las referidas al procedimiento de reexamen de las resoluciones del Tribunal General, cuya complejidad progresivamente se había manifestado al ponerlas en práctica. Este último procedimiento fue abreviado, por tanto, en el proyecto de Reglamento.

Con este mismo propósito - y en respuesta al deseo expresado por varios Estados miembros -, se asignó un título a todos los artículos del proyecto de Reglamento y dentro de cada artículo se numeraron todos los 
párrafos. En ciertos casos, esta operación obligó a fragmentar en artículos diferentes los textos existentes, para que cada artículo tuviera un objeto propio. Aunque esta fragmentación incrementó el número de artículos, pretendía hacer más legible el texto propuesto en su conjunto.

Y cuarto. Armonizar y racionalizar los términos empleados en las diferentes versiones lingüísticas del Reglamento de Procedimiento. A entender del Tribunal de Justicia, a cada realidad jurídica concreta debería corresponder un mismo y único término. Y ello porqué se había puesto de manifiesto que el Reglamento anteriormente vigente, al haber sido sometido a modificaciones sucesivas, empleaba a veces términos distintos para referirse a una misma realidad, lo que podía suscitar dudas sobre el auténtico objeto de las disposiciones en cuestión, mientras que, en ciertos casos aislados, resultaba evidente que el término utilizado no correspondía con la situación jurídica contemplada.

Cabe decir que los trabajos y trámites siguientes a la remisión del proyecto por parte del Tribunal de Justicia al Consejo de la Unión Europea hasta su definitiva adopción, no alteraron sustancial ni significativamente el texto inicial, dejando intactos, por lo tanto, el espíritu, los objetivos y la finalidad de la propuesta. Por este motivo, no nos referiremos a dichos trabajos y trámites 8 .

A continuación, y siguiendo por motivos prácticos la misma estructura del Reglamento de Procedimiento en vigor desde el día 1 de noviembre de 2012, vamos a intentar poner de manifiesto sus cambios y novedades más importantes respecto del texto que le precedía.

\section{La organización del Tribunal}

Como en el Reglamento de Procedimiento derogado, el Título Primero del nuevo Reglamento de Procedimiento se refiere a la organización del Tribunal. Este título - que reproduce el contenido de los dos primeros títulos del Estatuto del Tribunal de Justicia- pretende esencialmente precisar las atribuciones de los principales actores del Tribunal y formular las normas que regulan el funcionamiento del mismo, su régimen lingüístico y los principios y métodos utilizados para determinar la Sala que conoce de cada asunto9.

${ }^{8}$ Pueden consultarse los documentos más significativos en el registro público del Consejo de la Unión Europea http://register.consilium.europa.eu

9 Véase al respecto, sobre la base de la regulación anterior, TIZZANO, A. y IANNUCCELLI, P., «La organización y el funcionamiento del Tribunal de Justicia», en Tratado de Derecho y Políticas de la Unión Europea, tomo V (Sistema Jurisdiccional de la UE), Cizur 
Aunque se ha modificado el orden los capítulos con respecto al anterior Reglamento, son pocos los cambios que se han realizado en el propio contenido de las disposiciones del título primero. El nuevo texto reproduce lo esencial de las disposiciones vigentes hasta el 1 de noviembre de 2012, afinándolas. Sin embargo, por lo que respecta a los actores del procedimiento, conviene destacar algunas modificaciones importantes con respecto al régimen derogado.

La modificación del Protocolo sobre el Estatuto del Tribunal de Justicia y su anexo I, en agosto de $2012^{10}$, según la cual se introduce - entre otros aspectos - la figura de Vicepresidente, ha obligado en consecuencia a reflejarla en el Reglamento de Procedimiento, concretamente en el sentido de precisar el método de designación y las atribuciones de ese puesto ${ }^{11}$.

También como continuación de los cambios introducidos en el Estatuto del Tribunal, por los que se aumenta de trece a quince el número de Jueces que integran la Gran Sala, se precisan en el reciente Reglamento de Procedimiento las normas relativas a la determinación de la composición de las Salas y al procedimiento que debe seguirse en caso de impedimento de un Presidente o de otro miembro de una Sala ${ }^{12}$.

Por último, la nueva norma adjetiva simplifica las reglas relativas a la Secretaría y a los servicios del Tribunal de Justicia, dado que ciertas disposiciones del texto anterior, como las referentes al juramento de los funcionarios o a la organización de los servicios, parecían excesivamente detalladas o incluso carentes de relación con la finalidad esencial de un Reglamento de Procedimiento ${ }^{13}$.

Menor, 2012, pp. 113-154; AZOULAI, L., «The Court of Justice after the Treaty of Lisbon», en Common Market Law Review, 2010, núm. 3, pp. 709-728; LENAERTS, K., ARTS, D. y MASELIS, I., Procedural Law of the European Union, Londres, 2006 (reimpresión de 2011), pp. 3-32; y NÂOMÉ, Le renvoi préjudiciel..., op. cit., pp. 15-37.

10 Modificado por el Reglamento (UE, Euratom) núm. 741/2012 del Parlamento Europeo y del Consejo, de 11 de agosto de 2012 (DO L 228, de 23 de agosto de 2012, p. 1).

11 Son especialmente significativos en este sentido los artículos 8, 10 y 13 del RPTJ. La tarea esencial del Vicepresidente consistirá en secundar al Presidente en el ejercicio de sus funciones y reemplazarlo en caso de impedimento de éste o cuando la presidencia quedase vacante.

12 Tales cuestiones se tratan en la Sección 1, Capítulo Séptimo, del Título Primero, del RPTJ relativa a la composición de las formaciones del Tribunal, señalando el artículo 27.1 que la Gran Sala estará compuesta, para cada asunto, por el Presidente y el Vicepresidente del Tribunal, tres Presidentes de Salas de cinco Jueces, el Juez Ponente y el número de Jueces necesarios para alcanzar un total de quince, especificándose además en dicho artículo la forma de elección de estos últimos Jueces y de los tres Presidentes de Salas de cinco Jueces.

${ }^{13}$ Se regulan los aspectos relativos a la Secretaría en los artículos 18 a 22 del RPTJ, concretamente el nombramiento del Secretario y del Secretario adjunto, sus atribuciones su responsabilidad en la llevanza del Registro, así como la consulta del mismo y de las sentencias y los autos. 
Aunque sigue teniendo gran importancia para la organización de los procedimientos ante el Tribunal de Justicia, el capítulo relativo a los derechos y obligaciones de los agentes ha sido trasladado al principio del segundo título del Reglamento, relativo a las disposiciones de procedimiento comunes. A este respecto, la regulación procedimental se ajusta a la estructura del propio Estatuto, puesto que las normas relativas a la representación de las partes ante el Tribunal de Justicia figuran también en el Título Tercero de éste, relativo al «Procedimiento ante el Tribunal de Justicia» ${ }^{14}$.

Las disposiciones concernientes al régimen lingüístico no han sufrido alteraciones sustanciales ${ }^{15}$. El Capítulo Octavo del Reglamento en vigor concuerda, pues, con el capítulo sexto del texto derogado, con la excepción del artículo 37, apartado 2, que constituye una novedad. Pues, en efecto, con el fin de clarificar las normas aplicables, según el objetivo antes mencionado, el Tribunal de Justicia ha estimado preferible agrupar en un mismo capítulo todas las disposiciones relativas al régimen lingüístico - lo que explica la decisión de incluir, en el mencionado artículo, las disposiciones relativas a la lengua de procedimiento en los recursos de casación y en los procedimientos de reexamen - y añadir una disposición destinada a precisar en qué lengua deben presentarse las demandas o los recursos interpuestos en el marco de un asunto preexistente, tales como las demandas de interpretación de sentencia o de revisión o las solicitudes de tasación de costas. Tanto por la propia naturaleza de los procedimientos de que se trata - procedimientos accesorios con respecto a un procedimiento principal - como por la necesidad de defender los derechos de las partes en litigio, el nuevo Reglamento precisa que en dichos procedimientos debe utilizarse la lengua de la resolución a la que se refieran, sin perjuicio de las excepciones ya establecidas en el texto anterior ${ }^{16}$.

${ }^{14}$ En los artículos 43 a 47 del RPTJ bajo el epígrafe «De los derechos y obligaciones de los agentes, asesores y abogados», se tratan los privilegios, inmunidades y facilidades; la condición de representantes de las partes; el alzamiento de la inmunidad; la exclusión del procedimiento de los actores; así como una consideración a los profesores y partes del litigio principal.

15 Sobre la anterior regulación, este aspecto ha sido estudiado, entre otros, por GAUDISSART, M.A., «Le régime et la pratique linguistiques de la Cour de justice des Communautés européennes», en Langues et construction européenne, Bruselas, 2010, pp. 137-160; MCAULIFFE, K., «Enlargement at the European Court of Justice: Law, Language and Translation», en European Law Journal, 2008, núm. 6, pp. 806-818.

${ }^{16}$ Esta voluntad de regular con detalle y precisión la determinación de la lengua de procedimiento y el empleo de la misma, convierte los artículos 37 y 38 del RPTJ en unos de los más extensos del nuevo texto. 


\section{Las disposiciones de procedimiento comunes}

Como en el Reglamento de Procedimiento anterior, el Título Segundo está dedicado al desarrollo del procedimiento ante el Tribunal y a los diferentes modos en que éste tramita los asuntos ${ }^{17}$. A diferencia de la normativa anterior, no se otorga preeminencia a uno de los tipos de recurso, sino que se agrupan en un solo título las disposiciones de procedimiento comunes a todos los tipos de recurso, y se abordan las características específicas de cada uno de ellos en títulos separados. Con ello se ha querido garantizar una mayor facilidad de lectura del conjunto del texto, puesto que cada uno de los títulos puede leerse de manera autónoma, sin necesidad de recurrir a remisiones a las demás disposiciones del Reglamento.

En cuanto a su estructura, el Título Segundo del Reglamento sigue, a grandes rasgos, la del título equivalente del Reglamento anterior. Tras tres capítulos dedicados, respectivamente, a los derechos y obligaciones de los representantes de las partes, a las notificaciones y a los plazos, se exponen los diferentes modos en que el Tribunal puede tramitar asuntos y se determinan las normas aplicables a esta tramitación, desde la fase escrita del procedimiento hasta la terminación del mismo, pasando por el contenido del informe preliminar y la atribución de los asuntos a las Salas, las diligencias de ordenación del procedimiento y las de prueba y, por último, el desarrollo de la fase oral del procedimiento ${ }^{18}$.

En lo que respecta a la fase escrita del procedimiento, conviene destacar la inclusión de una disposición destinada a facultar al Tribunal para que determine, mediante decisión, las condiciones en las que la traducción de los escritos de alegaciones o de las observaciones presentados en un asunto podrá limitarse, por ser dichos textos excesivamente largos, a los pasajes esenciales de los mismos. Esta fórmula - que se inspira en la establecida en el artículo 104, apartado 1, del Reglamento de Procedimiento anterior para las peticiones de decisión prejudicial voluminosas - pretende salvaguardar la capacidad del Tribunal para resolver en un plazo razonable los litigios que le son sometidos, objetivo a veces difícil de cumplir cuando un recurrente en casación o una de las partes del litigio principal, no siempre conscientes

17 Para más detalle véase LENAERTS, ARTS y MASELIS: Procedural Law..., op. cit., pp. 523 y ss.; DÍAZ ABAD, N., «La defensa jurídica ante el Tribunal de Justicia de la Unión Europea», en Tratado de Derecho y Políticas..., op. cit., pp. 197-219; EDWARD, D., «How the Court of Justice Works», en European Law Journal, 1995, núm. 6, pp. 539-558; NAÔMÉ, Le renvoi préjudiciel..., op. cit., pp. 15-37; y especialmente MARTÍNEZ GIMENO, M., «Cuestiones procedimentales (I): Disposiciones comunes a todo procedimiento, recursos directos y cuestiones prejudiciales», Tratado de Derecho y Políticas..., op. cit., pp. 677-719.

18 El Título Segundo, que comprende un total de nueve capítulos, se extiende desde el artículo 43 al artículo 92 del RPTJ, y constituye el más amplio. 
del contexto linguíístico en el que el Tribunal trabaja, presentan ante el Tribunal un escrito de alegaciones de un centenar de páginas ${ }^{19}$.

En el capítulo relativo a las diligencias de ordenación del procedimiento y a las de prueba, el texto en vigor clarifica el tipo de diligencias que pueden acordar el propio Tribunal o la Sala que conoce del asunto ${ }^{20}$, al tiempo que simplifica las normas relativas al interrogatorio de testigos y a los dictámenes periciales ${ }^{21}$.

Por último se han introducido varias modificaciones en las normas que regulan la fase oral del procedimiento. Una de estas modificaciones consiste en la desaparición del informe para la vista. Al parecer, la redacción y la traducción de dicho informe consumían una buena parte de los recursos del Tribunal, sin que el informe constituyera una aportación de especial importancia para la tramitación del asunto, dado que se limitaba a reproducir los motivos o alegaciones invocados por las partes en sus escritos de alegaciones o en sus observaciones escritas. Se suprime, pues, la obligación de elaborar dicho informe, tal como ocurría ya, por lo demás, en los procedimientos acelerados o en los procedimientos de urgencia. En cambio, la importancia de las vistas en sí queda reforzada, puesto que, en la reforma realizada el Tribunal tomará la decisión de celebrar una vista cada vez que le pareciera necesario, y en particular cuando estime que las partes o los interesados mencionados en el artículo 23 del Estatuto no hayan podido exponer suficientemente su punto de vista en la fase escrita del procedimiento ${ }^{22}$.

19 Se trata del artículo 58 del RPTJ relativo a la longitud de los escritos procesales.

${ }^{20}$ Las diligencias de ordenación del procedimiento acordadas por el Tribunal se contemplan en el artículo 61, que constituye un artículo nuevo, que pretende recoger en el RPTJ las diligencias que con más frecuencia se proponen en ese informe, concretamente las preguntas del Tribunal a las partes para que respondan por escrito $\mathrm{u}$ oralmente o las propuestas de que concentren sus informes orales en puntos concretos. Por otra parte, el artículo 62 del RPTJ se refiere a las diligencias de ordenación acordadas por el Juez Ponente o el Abogado General y se basan en el artículo 54 bis del RPTJd, aunque se añade la posibilidad de que, con posterioridad al examen del asunto en reunión general, se remitan a las partes o a los interesados mencionados en el artículo 23 del Estatuto preguntas que deberían responderse en la vista. Este añadido corresponde a la práctica habitual y pone de manifiesto la voluntad de conseguir que la vista oral sea lo más útil e interactiva posible, comunicando por adelantado a las partes e interesados mencionados todas las preguntas que el Juez Ponente o el Abogado General pudieran plantearse al estudiar el expediente y que aún no hubieran sido comunicadas a estas partes o interesados al término de la reunión general.

21 Así, por ejemplo, en cuanto los gastos relacionados con los interrogatorios de testigos y dictámenes periciales, se contemplan solamente en el artículo 73 del RPTJ, agrupando así disposiciones que se encontraban dispersas en el RPTJd.

${ }^{22} \mathrm{Si}$ bien se aporta a este respecto una precisión adicional en el artículo 76.3 del RPTJ que no existía en el RPTJd la realidad es que la misma concuerda en buena medida con la práctica habitual del Tribunal, en el sentido de resolver sin haber celebrado antes una vista oral, por ejemplo sobre la base de lo dispuesto en el artículo 104.3 del RPTJd, o cuando la so- 
El nuevo Reglamento codifica, por otra parte, varias normas que tienen su origen en la práctica del Tribunal o en su jurisprudencia, tales como la posibilidad de organizar vistas comunes a varios asuntos cuando las similitudes existentes entre ellos así lo permitan, o la clarificación de las circunstancias que pueden dar lugar a la apertura o a la reapertura de la fase oral del procedimiento. También se recoge la posibilidad de que el Presidente conceda a una de las partes que han participado en el procedimiento el derecho a escuchar la grabación de las palabras pronunciadas en la vista oral, en su versión original ${ }^{23}$.

Este título se cierra con un capítulo sobre el contenido de las sentencias y autos del Tribunal, la fecha a partir de la cual adquieren obligatoriedad y la publicidad que deben recibir ${ }^{24}$.

\section{Las cuestiones prejudiciales}

Como ya señalábamos al principio del presente trabajo, en el derogado Reglamento de Procedimiento las cuestiones prejudiciales ocupaban una posición marginal. Redactado en una época en la que la preocupación esencial era el control de la legalidad de la actuación de las instituciones recién creadas, el Reglamento de Procedimiento dedicaba lógicamente lo esencial de sus disposiciones a los recursos directos, mientras que la cuestión prejudicial, muy poco conocida en 1953, se trataba en él como un procedimiento especial ${ }^{25}$, al igual que la suspensión de la ejecución o la intervención, y del mismo modo, incluso, que los recursos extraordinarios, como la oposición de tercero o la revisión de sentencias.

Ahora bien, aunque es innegable que durante mucho tiempo las cuestiones prejudiciales han sido menos numerosas que los recursos de anulación, de omisión o de incumplimiento interpuestos ante el Tribunal, actualmente

licitud de celebración de la misma no está motivada, al igual que decide a veces organizar una vista oral aunque ninguna de las partes lo haya solicitado. De hecho, el artículo 76 del RPTJ en su conjunto recalca con más intensidad el criterio decisivo para la celebración o no de una vista, concretamente según el valor añadido que la misma puede aportar a la tramitación del asunto. En cualquier caso se organizará una vista si el Tribunal estima que las partes o los interesados no han podido exponer suficientemente su punto de vista en la fase escrita del procedimiento.

${ }^{23}$ Nos referimos en concreto a los artículos 77, 83 y 85 del RPTJ.

${ }^{24}$ El Capítulo Noveno, «De las sentencias y los autos», se extiende desde el artículo 86 al 92 del RPTJ, si bien no presenta novedades significativas.

${ }^{25}$ Las cuestiones prejudiciales y otros procedimientos en materia de interpretación se trataban en el Título Tercero del RPTJd, bajo el epígrafe «De los procedimientos especiales», concretamente en el Capítulo Noveno, artículos 103 y 104, a los que posteriormente se tuvieron que añadir los artículos 104 bis y 104 ter. 
la situación ha cambiado, como consecuencia de las reformas estructurales de la institución y de las sucesivas ampliaciones de la Unión Europea. Las cuestiones prejudiciales constituyen hoy, con diferencia, la principal categoría entre los asuntos sometidos al Tribunal y desempeñan un papel decisivo, desde varios puntos de vista, en el desarrollo del Derecho de la Unión y en la integración de éste en los ordenamientos jurídicos nacionales ${ }^{26}$. Por lo tanto, se ha estimado oportuno desplazar las disposiciones que el Reglamento de Procedimiento les dedica, colocándolas inmediatamente después de las disposiciones de procedimiento comunes, y clarificar, en beneficio de las partes y de los tribunales nacionales, ciertas normas derivadas de la jurisprudencia o inherentes a la propia naturaleza del procedimiento prejudicial $^{27}$.

Tras recordar brevemente el ámbito de aplicación de este procedimiento y el contenido indispensable de toda petición de decisión prejudicial ${ }^{28}$, el nuevo Reglamento reproduce así el contenido del artículo 23 del Estatuto, enumerando las partes autorizadas a presentar observaciones escritas u ora-

26 Dada la relevancia de la cuestión prejudicial en la formación del Derecho de la Unión Europea, su aspecto procedimental ha sido extensamente tratado por la literatura jurídica, siendo de destacar, entre muchos otros, los trabajos de BROBERG, M. y FENGER, N., Preliminary References to the European Court of Justice, New York, 2010, pp. 340-399; LENAERTS, ARTS y MASELIS, Procedural Law..., op. cit., pp. 608-616; NAÔMÉ, Le renvoi préjudiciel..., op. cit., pp. 297-318; CLERGERIE, J. L., Le renvoi préjudiciel, París, 2000, pp. 92-121. VANDERSANDEN, G., Renvoi préjudiciel en droit européen, Bruselas, 2013, pp. 101-163; PERTEK, J., Coopération entre juges nationaux et Cour de justice de l'UE. Le renvoi préjudiciel, Bruselas 2013, pp. 133-154; CIENFUEGOS MATEO, M., «Juez Nacional-Tribunal de Justicia: la cuestión prejudicial», en Tratado de Derecho y Políticas..., op. cit., pp. 599-607; del mismo autor «El planteamiento de cuestiones prejudiciales por los órganos jurisdiccionales españoles: teoría y práctica», en España ante los Tribunales internacionales europeos. Cuestiones de política judicial, Oñati, 2008, pp. 47-101; MARTÍNEZ GIMENO, «Cuestiones procedimentales (I)...», Tratado de Derecho y Políticas..., op. cit., pp. 759-779; y también los clásicos JIMENO BULNES, M., La cuestión prejudicial del artículo 177 TCE, Barcelona, 1996, pp. 401-453; VILLAGÓMEZ CEBRIÁN, M., La cuestión prejudicial en el Derecho comunitario europeo, 1994, pp. 115-133; y SILVA DE LAPUERTA, R., El procedimiento ante el Tribunal de Justicia de las Comunidades Europeas, Madrid, 1993, pp. 254-263.

${ }^{27}$ Se dedica íntegramente el Título Tercero del RPTJ a las cuestiones prejudiciales.

${ }^{28}$ El artículo 93 del RPTJ, relativo al ámbito de aplicación de las cuestiones prejudiciales, concuerda en lo esencial con los apartados 1 y 2 del artículo 103 del RPTJd, aunque de forma más simplificada, habida cuenta de la revalorización de las cuestiones prejudiciales que lleva a cabo el nuevo texto. En cambio, desaparecen las referencias a los Protocolos específicos mencionados en el artículo 103.2 del RPTJ, dada la evolución de competencias de la Unión y, en particular, de la integración en el Derecho de la Unión de materias que antes no regulaba. Y el artículo 94 del RPTJ, concerniente al contenido de la petición de decisión prejudicial, aparece como una nueva disposición que se inspira directamente en la Nota informativa sobre el planteamiento de cuestiones prejudiciales por los órganos jurisdiccionales nacionales (DO C 160 de 25 de mayo de 2011, pp. 1-4). 
les ante el Tribunal ${ }^{29}$ y delimitando con mayor claridad, por remisión a las normas de procedimiento nacionales, el concepto de «partes del litigio principal» y las consecuencias para el procedimiento ante el Tribunal de Justicia de la admisión por el órgano jurisdiccional remitente de una nueva parte en el litigio ${ }^{30}$.

Por la voluntad de preservar, en determinadas circunstancias, el anonimato de las personas implicadas en litigios concretos, el nuevo texto incluye una disposición que le faculta para, de oficio o a instancia de parte, no divulgar en la información accesible al público el nombre de una o varias personas o los datos que permitan identificarlas. Aunque no está destinada a aplicarse a todo tipo de asuntos, esta disposición reviste sin embargo especial importancia en un momento en que se someten al Tribunal a un ritmo creciente litigios de carácter penal o en los que se discute el ejercicio de la autoridad parental o la guarda de hijos menores $^{31}$.

Con un mismo objetivo de protección de los intereses de las partes del litigio principal, y en particular de las partes carentes de recursos económicos, se clarifican por lo demás los requisitos para la concesión de asistencia jurídica gratuita, en particular en los supuestos en que el órgano jurisdiccional remitente haya otorgado ya dicha asistencia, y formula las normas indispensables para examinar con rapidez las solicitudes de asistencia jurídica gratuita. Así, tan pronto como se presenten en la Secretaría, estas solicitu-

${ }^{29}$ Así el artículo 96 del RPTJ, que carece de equivalente en el RPTJd tiene un doble objetivo: por una parte, enumerar, para mayor claridad, a todas las personas o entidades autorizadas a presentar observaciones ante el Tribunal; y, por otra parte, recordar que la presentación de observaciones escritas tiene carácter facultativo y que el hecho de renunciar a presentarlas no afecta a la participación en la fase oral del procedimiento. Es decir, resulta perfectamente lícito que una parte del litigio principal o un Estado miembro que no ha presentado observaciones escritas formulen observaciones en la vista oral, si esta tiene lugar.

${ }^{30} \mathrm{El}$ artículo 97 del RPTJ, que trata esos aspectos, no es totalmente nuevo, pues recoge partes del artículo 104 del RPTJd, pero se completa consignando la práctica consistente en tomar contacto con el órgano jurisdiccional nacional remitente en caso de duda, a fin de obtener más precisiones sobre las normas nacionales en materia de representación y comparecencia de las partes. No obstante, sí aparecen como novedad los puntos 1 y 2 de dicho artículo 97 del RPTJ, en los que se pone de manifiesto el interés del Tribunal en no permitir que la tramitación del asunto se retrase a causa de intervenciones múltiples en el transcurso del procedimiento y su voluntad de no sobrepasar los límites trazados por el órgano jurisdiccional que le sometió el asunto.

${ }^{31}$ Obviamente para ser eficaz la posibilidad que ofrece el artículo 98 del RPTJ debe adoptarse en las fases iniciales de la tramitación del asunto, pues dado el desarrollo de las tecnologías de la información, y en particular de internet, de una decisión de anonimizar adoptada después de la publicación de la comunicación relativa a la petición de decisión prejudicial en el DO tendría poco sentido. 
des serán atribuidas al Juez encargado del asunto en el que son formuladas y la decisión al respecto será adoptada, en breve plazo, por la Sala más pequeña a la que dicho Juez esté adscrito ${ }^{32}$.

Tomando en consideración la experiencia adquirida en la aplicación de los procedimientos prejudiciales y, en particular, de las dificultades provocadas por la interposición de recursos, a nivel nacional, contra peticiones de decisión prejudicial o por la retirada de tales peticiones en una fase muy avanzada del procedimiento ante el Tribunal de Justicia, el nuevo Reglamento contiene igualmente una disposición que precisa mejor las circunstancias en que se le atribuye el conocimiento del asunto ${ }^{33}$.

Por último, se introducen diversas modificaciones al respecto de la anterior norma adjetiva, con objeto, por una parte, de ofrecer al Tribunal los medios necesarios para tramitar con mayor rapidez aún los asuntos que le son sometidos y, por otra, de colmar algunas lagunas aparecidas en la aplicación de los procedimientos acelerados y de los procedimientos de urgencia.

En cuanto al primer tipo de medidas, merece la pena mencionar la simplificación del procedimiento establecido para la adopción por el Tribunal de un auto motivado, cuando la respuesta a la cuestión prejudicial no suscite ninguna duda razonable ${ }^{34}$, o la posibilidad de que tanto el Tribunal como las partes se comuniquen escritos procesales por fax o cualquier otro medio técnico de comunicación en los procedimientos acelerados, sin perjuicio de la transmisión posterior del original ${ }^{35}$.

Respecto a la segunda serie de modificaciones, cabe mencionar, en particular, la posibilidad de que el Presidente del Tribunal abra de oficio un procedimiento acelerado - posibilidad que ya existía para los procedimientos prejudiciales de urgencia-, o la facultad del Tribunal de Justicia, en los procedimientos prejudiciales de urgencia, de instar a un Estado miembro distinto del Estado del que depende el órgano jurisdiccional remitente a que

${ }^{32}$ Se contienen estas reglas en el Capítulo Cuarto del Título Tercero, artículos 115 a 118 del RPTJ. En efecto, el RPTJd que regulaba con cierta extensión la posibilidad de otorgar asistencia jurídica gratuita en los recursos directos, era extremadamente conciso en lo que respecta a los procedimientos prejudiciales, de tal forma que con las nuevas disposiciones se intenta colmar esta laguna, ajustándola por otra parte a la Carta de los Derechos Fundamentales de la Unión Europea, que en su artículo 47 alude a la prestación de asistencia jurídica gratuita a quienes no dispongan de recursos suficientes.

33 Se establece en el artículo 100 del RPTJ.

${ }^{34}$ Así aparece en el artículo 99 del RPTJ, que en parte se inspira en el artículo 104.3 del RPTJd.

${ }^{35}$ La referida tramitación de escritos procesales se regula en el artículo 106 del RPTJ al respecto de los procedimientos acelerados y en el artículo 114 del RPTJ al respecto de los procedimientos de urgencia, si bien este último artículo parece superfluo toda vez que remite íntegramente al 106 del RPTJ. 
presente observaciones escritas o responda por escrito a ciertas preguntas que se han planteado al tramitar el asunto ${ }^{36}$.

\section{Los recursos directos}

Los recursos directos constituyen, en cifras, el segundo grupo de asuntos sometidos al Tribunal de Justicia y son esencialmente recursos por incumplimiento de Estados miembros ${ }^{37}$. Lógicamente, el nuevo Reglamento se ocupa de ellos inmediatamente después de las cuestiones prejudiciales, en su Título Cuarto. El referido título reproduce y precisa algunas disposiciones del texto derogado ${ }^{38}$, pero contiene varias innovaciones.

Entre ellas, conviene destacar el establecimiento de un plazo de dos meses para la presentación del escrito de contestación a la demanda, respecto del anterior plazo de un mes ${ }^{39}$; la simplificación de la invocación de motivos nuevos en el curso del proceso ${ }^{40}$; y la atribución al Presidente de fijar los plazos en que se presentarán los escritos procesales de réplica y de dúplica, precisando, en su caso, las cuestiones de Derecho sobre las que debe-

36 Se trata de los artículos 105.1 y 109.3 del RPTJ. En efecto, desde la entrada en vigor del procedimiento de urgencia, el 1 de marzo de 2008, se ha observado que ciertos asuntos, tales como los relativos a la interpretación de la Decisión Marco del Consejo, de 13 de junio de 2002, relativa a la orden de detención europea y a los procedimientos de entrega entre Estados miembros (DO L 190 de 18 de julio de 2002, p. 1), o del Reglamento (CE) núm. 2201/2003 del Consejo, de 27 de noviembre de 2003, relativo a la competencia, el reconocimiento y la ejecución de resoluciones judiciales en materia matrimonial y de responsabilidad parental (DO L 338 de 23 de diciembre de 2003, p. 1), afectan con frecuencia a los intereses de dos o más Estados miembros. Por esta razón, se amplía el círculo de personas y entidades autorizadas a presentar observaciones escritas en un procedimiento prejudicial de urgencia, en la medida en que el buen desarrollo del procedimiento lo permita.

37 Véase la nota 5.

$38 \mathrm{Al}$ respecto de la normativa derogada, véase LENAERTS, ARTS y MASELIS, Procedural Law..., op. cit., pp. 523-607; MARTÍNEZ GIMENO, «Cuestiones procedimentales (I)...», Tratado de Derecho y Políticas..., op. cit., pp. 719-759.

39 Tal novedad aparece en el artículo 124 del RPTJ. En efecto, el anterior plazo de un mes se revelaba a veces insuficiente para que el demandado preparara adecuadamente su defensa, en particular en el caso de los recursos por incumplimiento, dada la necesidad de consultar a diferentes agentes y a diversos ministerios a fin de reunir los datos indispensables para redactar el escrito de contestación. Ello motivaba que algunas partes presentaran sistemáticamente solicitudes de prórroga de dicho plazo, creando así una carga de trabajo adicional tanto para el Tribunal como para ellas. El nuevo precepto, en su apartado 3, califica de excepcionales las prórrogas de dicho plazo y, por lo tanto, deberán constar debidamente motivadas.

40 La encontramos en el artículo 127 del RPTJ, que no obstante reproduce el contenido del artículo 42, apartado 2, del RPTJd. 
rán versar ${ }^{41}$; así como la posibilidad de que el Presidente decida de oficio la tramitación de un asunto a un procedimiento acelerado ${ }^{42}$.

Por último, aunque el nuevo texto no ha modificado el Reglamento anterior en lo relativo a las costas del procedimiento, exceptuando una precisión sobre la Sala competente para resolver en caso de discrepancia sobre las costas recuperables ${ }^{43}$, agrupa en cambio en un único capítulo toda una serie de disposiciones, antes dispersas en cuatro capítulos distintos, sobre las demandas y recursos relativos a las sentencias y autos del Tribunal (rectificación, omisión de pronunciamiento, oposición a la sentencia, oposición de tercero, interpretación y revisión) $)^{44}$.

Ninguna modificación sustancial se introduce, en cambio, en el capítulo relativo a la suspensión y a las demás medidas provisionales.

\section{Los recursos de casación contra las resoluciones del Tribunal General}

En lo que respecta al tercer - y último - grupo importante de asuntos de entre los que se someten al Tribunal de Justicia, a saber, los recursos de

${ }^{41}$ Ello se explica por el interés del Tribunal de conseguir que las fases escrita y oral del procedimiento sean lo más útiles posible. De esta forma, si el Tribunal ya ha identificado claramente los puntos esenciales del asunto tras el primer intercambio de escritos procesales, podrá instar a las partes a concentrarse en ellos, lo que debe permitir, por un lado, que las partes eviten desarrollar en la réplica y en la dúplica puntos sobre los que el Tribunal estima disponer de información suficiente, favoreciendo así una tramitación más rápida del asunto, ya que en el segundo intercambio de escritos procesales sólo se trataran las cuestiones aún abiertas.

${ }^{42}$ La novedad se recoge en el artículo 133 del RPTJ. Las razones por las que se incluye esta posibilidad son idénticas, mutatis mutandi, a las que han llevado a añadirla en los procedimientos prejudiciales.

${ }^{43}$ El artículo 145 del RPTJ, relativo a las solicitudes de tasación de costas, se distingue en dos puntos del artículo 74 del RPTJd. Dispone así, por una parte, que tales solicitudes serán examinadas por la formación más pequeña a la que esté adscrito el Juez Ponente, es decir, por una Sala de tres o de cinco Jueces, según los casos, lo que permitirá en particular evitar movilizar a trece o veintisiete Jueces en los casos en que el asunto al que se refiera la solicitud hubiera sido atribuido a la Gran Sala o al Pleno. Por otra parte, como tales solicitudes no plantean por lo demás ninguna cuestión de Derecho nueva, dicho artículo elimina la presentación de conclusiones del Abogado General en este supuesto, y la Sala se limitará a oír su opinión antes de pronunciarse.

${ }^{44}$ Se trata del Capítulo Noveno, «De las demandas y recursos relativos a las sentencias y autos», artículos 153 a 159 del RPTJ. Reflejando la voluntad del Tribunal de tratar con rapidez las demandas de esta índole, el RPTJ dispone por otra parte que serán encomendadas automáticamente al Juez Ponente encargado del asunto con el que la demanda o el recurso estén relacionados y atribuidas a la Sala que se pronunció sobre dicho asunto, y la única excepción a esta atribución automática se explica tanto por la duración del plazo en el que puede presentarse una demanda de revisión como por la especial naturaleza de dicho procedimiento. 
casación interpuestos contra las resoluciones del Tribunal General, el nuevo texto reproduce, en lo esencial, las disposiciones del anterior Reglamento de Procedimiento, pero aumentando su extensión, tanto por ánimo de alinearlas con las disposiciones del Estatuto relativas a los requisitos de fondo y de forma del recurso de casación como por interés en aclarar la auténtica naturaleza de este tipo de recursos, y en particular la articulación entre el recurso de casación y la adhesión a la casación ${ }^{45}$.

Entre las novedades del Reglamento, conviene mencionar en primer lugar el refuerzo de los requisitos exigidos para la presentación de un recurso de casación o de una adhesión a la casación. Tanto en un caso como en otro, se precisa, en efecto, que las pretensiones deben tener por objeto la anulación, total o parcial, de la resolución del Tribunal General tal y como figura en el fallo de dicha resolución. Con esta precisión, que es consecuencia de la experiencia adquirida en la aplicación de este procedimiento, veinte años después de la creación del Tribunal General, se pretende sobre todo prevenir la presentación de recursos de casación o de adhesiones a la casación que tengan por único objeto impugnar un aspecto concreto del razonamiento seguido por el Tribunal General. Así, si una parte ha visto estimadas sus pretensiones en el proceso ante el Tribunal General, no estará facultada para interponer recurso de casación contra su resolución, sin que esto afecte, no obstante, a la posibilidad de que una parte impugne, en su adhesión a la casación, la decisión expresa o implícita de este último Tribunal sobre la admisibilidad del recurso interpuesto ante él ${ }^{46}$.

Con la intención de no prolongar inútilmente la tramitación de los recursos de casación y teniendo en cuenta la especial naturaleza de este tipo de asuntos, se endurecen los requisitos necesarios para que el recurso de casación y el escrito de contestación puedan completarse con un escrito de réplica y un escrito de dúplica. Para presentar estos últimos escritos será pre-

45 En el RPTJd este específico recurso se localizaba en el Título Cuarto, artículos 110-123, mientras que en el RPTJ su régimen se encuentra en el Título Quinto, artículos 167-190. LENAERTS, ARTS y MASELIS, Procedural Law..., op. cit., pp. 452-471 y 617-621. CASTILLO DE LA TORRE, F., «Cuestiones procedimentales (II): Recurso de casación, medidas provisionales y recursos extraordinarios», en Tratado de Derecho y Políticas..., op. cit., pp. 800-812.

46 Así lo encontramos en los artículos 169 y 178 del RPTJ, relativos, respectivamente, a las pretensiones, motivos y alegaciones del recurso o de la adhesión a la casación. Tanto en uno como en otro artículo se añade el requisito, ampliamente desarrollado por la jurisprudencia, con arreglo al cual el recurrente o los adherentes deben identificar con precisión los extremos de la sentencia o auto que impugnan, de tal forma que no pueden limitarse a impugnar dicha resolución en términos genéricos, sin indicar el error o errores de Derecho cometidos a su juicio por el Tribunal General. Así, entre otras, las Sentencias de 4 de julio de 2000, Bergaderm y Goupil/Comisión, C-352/98 P, Rec. pp. I-5291, ap. 34-35; de 30 de septiembre de 2003, Eurocoton y otros/Consejo, C-76/01 P, Rec. pp. I-10091, ap. 46-47; de 7 de julio de 2005, Le Pen/Parlamento, C-208/03 P, Rec. pp. I-6051, ap. 39. 
ciso, por un lado, que la parte recurrente haya presentado una petición de réplica debidamente motivada en un plazo de siete días a partir de la notificación del escrito de contestación y, por otro lado, que el Presidente del Tribunal de Justicia estime necesaria dicha réplica, tras haberlo consultado con el Juez Ponente y con el Abogado General encargado del asunto. En particular, puede autorizarse la presentación de dichos escritos a fin de permitir que la parte recurrente se pronuncie sobre una excepción de inadmisibilidad o sobre nuevas circunstancias invocadas en el escrito de contestación. Reflejando la práctica del Tribunal, el texto en vigor precisa sin embargo que, en el caso de que estime esta petición, el Presidente podrá instar a las partes a limitar el número de páginas y el objeto de los escritos de réplica y de dúplica ${ }^{47}$.

Además de añadir estas precisiones, el nuevo Reglamento confirma la posibilidad de que las partes en el procedimiento ante el Tribunal General se adhieran a la casación, impugnando igualmente la resolución contra la que se dirige el recurso de casación. Tanto por afán de claridad como para facilitar la tramitación de la adhesión a la casación, se precisa sin embargo que la adhesión a la casación debe formalizarse en un escrito separado, distinto del escrito de contestación ${ }^{48}$. Por lo demás, el nuevo texto infiere las consecuencias pertinentes del carácter accesorio de la adhesión a la casación, al disponer que esta última perderá su objeto si el recurrente desiste de su recurso de casación o cuando dicho recurso se declare manifiestamente inadmisible ${ }^{49}$.

En el capítulo sobre los recursos de casación se recoge la posibilidad de que el Tribunal de Justicia declare el recurso de casación o la adhesión a la casación manifiestamente fundados mediante un auto que haga referencia a la jurisprudencia pertinente. Esta posibilidad pretende permitir que el Tribunal de Justicia ofrezca una solución rápida al litigio cuando tal solución no suscite ninguna duda, habida cuenta de las cuestiones de Derecho resueltas por el Tribunal de Justicia en asuntos anteriores, sin otra diferencia que la identidad del recurrente en casación o de la parte que se adhirió a la casación ${ }^{50}$.

47 Se dispone así en el artículo 175 del RPTJ.

${ }^{48}$ De acuerdo con lo dispuesto en el artículo 176.1 del RPTJ, la adhesión a la casación debe formularse dentro del plazo establecido para la presentación del escrito de contestación, esto es de dos meses.

${ }^{49} \mathrm{El}$ artículo 183 del RPTJ constituye una novedad destacable al respecto del texto anterior.

${ }^{50}$ Ello se establece en el artículo 182 del RPTJ. Cabe decir que esta posibilidad la encontramos también en el artículo 99 del RPTJ (art. 104.3 del RPTJd), según el cual se faculta al Tribunal de Justicia para resolver mediante auto motivado en materia prejudicial cuando la respuesta a la cuestión planteada por el órgano jurisdiccional remitente sea idéntica a otra sobre la que el Tribunal de Justicia ya haya resuelto, pueda deducirse claramente de la jurisprudencia o no suscite ninguna duda razonable. 
Por último, y como en otros títulos, se aclaran y detallan las normas relativas a las costas y a la asistencia jurídica gratuita en los recursos de casación ${ }^{51}$.

\section{El reexamen de las resoluciones del Tribunal General}

Aunque el procedimiento de reexamen raramente se ha aplicado hasta ahora, a causa de su propia naturaleza y de la gravedad del perjuicio que lo justifica - recordemos que se trata de un procedimiento iniciado por el primer Abogado General cuando considere que existe un riesgo grave de que se vulnere la unidad o la coherencia del Derecho de la Unión-, dicho procedimiento había sido sin embargo criticado por su pesadez y su complejidad, así como por la falta de claridad en lo relativo a las condiciones en que podía iniciarse. Así, existían dudas en cuanto al momento concreto de transmisión al Tribunal de Justicia de los autos del procedimiento ante el Tribunal General, mientras que el hecho de que el asunto fuera examinado por dos Salas distintas del Tribunal de Justicia - una de las cuales se pronunciaba sobre la propuesta de reexamen del primer Abogado General y la otra sobre las cuestiones objeto de reexamen, habida cuenta de las observaciones presentadas por las partes y demás interesados - podía suscitar interrogantes, tanto por los recursos humanos y materiales que debía movilizar como por las eventuales divergencias de opinión que pudieran aparecer entre ambas Salas ${ }^{52}$.

En el nuevo Reglamento, se simplifica pues el procedimiento de reexamen $^{53}$, corrigiendo en ciertos aspectos el régimen derogado y estableciendo un régimen distinto entre el reexamen de las resoluciones adoptadas en casación por el Tribunal General y el reexamen de las resoluciones que dicho Tribunal podría eventualmente adoptar en caso de cuestiones prejudiciales, aunque el procedimiento seguido en uno y otro caso sería esencialmente idéntico ${ }^{54}$. Por una parte, se establece que se designará una Sala de cinco Jueces encargada de examinar, durante un período de un año y en las condiciones que establece el Reglamento de Procedimiento, si procede reexaminar una resolución del Tribunal General conforme a la propuesta en ese sen-

51 Se trata del Capítulo Séptimo, del Título Quinto, artículos 184-189, del RPTJ.

52 FARDET, C., «Le "réexamen" des décisions du Tribunal de première instance«, Revue du marche commun et de l'Union Européenne, 2004, núm. 476, pp. 184-193. LENAERTS, ARTS y MASELIS, Procedural Law..., op. cit., pp. 452-471. CASTILLO DE LA TORRE, «Cuestiones procedimentales (II)...», en Tratado de Derecho y Políticas..., op. cit., pp. 818-821.

53 La regulación se efectúa en el Título Sexto, artículos 191-195, del RPTJ.

${ }^{54}$ La distinción de régimen se realiza en los artículos 193 y 194 del RPTJ. 
tido formulada por el primer Abogado General. En este sentido, el nuevo texto se inspira en el modelo aplicado con éxito a la tramitación de los asuntos sometidos al procedimiento prejudicial de urgencia ${ }^{55}$.

Por otra parte, la norma adjetiva en vigor suprime la regla relativa a la designación de un nuevo Juez Ponente en el caso de que se inicie efectivamente el procedimiento de reexamen. Por lo tanto, es una sola y misma Sala - la Sala que se ocupe ese año de los procedimientos de reexamen - la que se pronunciará tanto sobre la propuesta de reexamen del primer Abogado General en un asunto concreto como sobre el fondo del asunto, es decir, sobre las cuestiones objeto de reexamen ${ }^{56}$.

Por último se clarifica la información y la remisión de documentación en los procedimientos de reexamen, al disponer que los autos del procedimiento del Tribunal General se remitirán a la Secretaría del Tribunal de Justicia tan pronto como se dicte o se firme la resolución del Tribunal General y que en el Diario Oficial de la Unión Europea se publicará un anuncio que indicará la fecha de la decisión de reexaminar la resolución del Tribunal General y las cuestiones que serán objeto de reexamen ${ }^{57}$.

\section{Los dictámenes}

Aunque el nuevo Reglamento no modifica ni el contenido esencial del procedimiento de dictamen ${ }^{58}$ ni su desarrollo, introduce sin embargo algu-

55 Con el fin de aligerar la carga de trabajo del Presidente del Tribunal de Justicia y de los Presidentes de Salas de cinco Jueces, el artículo 191 del RPTJ, abandona así el mecanismo del artículo 123 ter del RPTJd.

56 Si bien el artículo 193 del RPTJ, relativo al reexamen de las resoluciones adoptadas en casación, y el artículo 194 del RPTJ, referente al reexamen de decisiones prejudiciales, son idénticos, en este último se añade un apartado adicional, que ya figuraba en el artículo 123 quinto del RPTJd, en el que se establece la obligación de informar al Tribunal General, al órgano jurisdiccional remitente, a las partes del litigio principal y a los demás interesados contemplados en el artículo 62 bis, párrafo segundo, del Estatuto, de la decisión de reexaminar o no la resolución del Tribunal General.

57 Tales aspectos también se acometen en los extensos artículos 193 y 194 del RPTJ.

58 Para más detalle: DÍEZ DE VELASCO, M., «La competencia consultiva del Tribunal de Justicia de las Comunidades Europeas», en El Derecho Comunitario Europeo y su aplicación judicial, Madrid, 1993, pp. 573-594; FRUTOS MIRANDA, J. y ESPÓSITO, C., «La función consultiva: los dictámenes previos a la conclusión de acuerdos internacionales», en Tratado de Derecho y Políticas..., op. cit., pp. 509-548; KOVAR, R., «La compétence consultative de la Cour de Justice et la procédure de conclusion des accords internationaux par la communauté économique européenne», en Mélanges offerts à P. Reuter, París, 1981, pp. 357-377; SINAGRA, A., «La funzione consultiva della Corte di Giustizia ex art. 228 del Trattato istitutivo della Comunità Economica Europea», en Studi di Diritto Europeo in onore di R. Monaco, Milan, 1977, pp. 695-719; LENAERTS, ARTS y MASELIS: Procedural Law..., op. cit., pp. 408-415. 
nas modificaciones con respecto al texto anterior que conviene mencionar ${ }^{59}$.

En primer lugar, en lo que respecta a los participantes en este procedimiento en el seno del Tribunal de Justicia, se dispone que a partir de ahora la solicitud de dictamen será atribuida a un solo Abogado General, y no a los Abogados Generales en conjunto. Con ello se pretende aligerar la carga de trabajo de los Abogados Generales y facilitar la toma de decisiones. En efecto, aunque la participación del conjunto de Abogados Generales podía explicarse en un momento en que la Comunidad iniciaba su andadura, tal participación parece hoy desmesurada, habida cuenta tanto del número de Abogados Generales como del hecho de que otros asuntos que plantean importantes cuestiones de principio son atribuidos a un solo Abogado General, incluso en el caso de que sean examinados por el Pleno ${ }^{60}$.

El Reglamento en vigor codifica a continuación la práctica seguida hasta ahora en la mayoría de los procedimientos de dictamen que se han ido abriendo, y dispone así, por una parte, que las solicitudes de dictámenes serán notificadas sistemáticamente a los Estados miembros, al Parlamento Europeo, al Consejo y a la Comisión Europea ${ }^{61}$ y, por otra parte, que el Tribunal podrá decidir celebrar una vista oral si lo estima necesario para emitir su dictamen ${ }^{62}$.

También se suprime toda referencia a las deliberaciones con carácter reservado, utilizando las sentencias como modelo para la declaración de terminación de los procedimientos de dictamen. Así, como ocurre con las sentencias, los dictámenes se emitirían en audiencia pública ${ }^{63}$.

\section{Los procedimientos especiales}

El nuevo Reglamento agrupa en un último título varios procedimientos especiales, aplicados en muy escasas ocasiones, y que anteriormente se encontraban dispersos en varios títulos y capítulos. No se modifica el contenido esencial de tales procedimientos, pero conviene mencionar la supresión

59 La nueva regulación se encuentra en el Título Séptimo, artículos 196 a 200 del RPTJ.

${ }^{60}$ Se establece esta modificación en el artículo 197 del RPTJ.

${ }^{61} \mathrm{Si}$ bien el artículo 196 del RPTJ viene a reproducir el contenido del artículo 107 del RPTJd, se ha simplificado ligeramente.

62 Se trata de una novedad contenida en el artículo 200 del RPTJ, aunque, no obstante, se trata de una facultad que el Tribunal ha utilizado tradicionalmente, puesto que en la mayoría de los asuntos que se le han sometido con arreglo al artículo 218 TFUE, apartado, 11, se ha celebrado una vista.

${ }^{63}$ El nuevo artículo 200 del RPTJ concuerda en esencia con el artículo 108, apartado 3, del RPTJd pero se completa disponiendo que el dictamen se emitirá en audiencia pública. 
del precepto, nunca aplicado, que facultaba a los órganos jurisdiccionales de Estados terceros partes en el acuerdo EEE de plantear una cuestión prejudicial al Tribunal de Justicia ${ }^{64}$, y la inclusión de una nueva disposición destinada a precisar, a raíz de la entrada en vigor del Tratado de Lisboa, los aspectos esenciales del procedimiento que debería seguirse en el caso de que se presentara al Tribunal una petición basada en el artículo 269 TFUE $^{65}$.

\section{Consideraciones finales}

Sin lugar a dudas, la modificación del Reglamento del Tribunal de Justicia deviene, no solamente de la voluntad de la propia institución de adaptarse a la realidad de los asuntos que conoce, sino de las exigencias impuestas por la entrada en vigor del Tratado de Lisboa en diciembre de 2009, por la modificación del Protocolo de su Estatuto en agosto de 2012 o, inclusive, de la necesidad de dar una mayor efectividad a las disposiciones que en materia de justicia se establecen en la Carta de los Derechos Fundamentales de la Unión Europea.

Es evidente que, por ahora, resulta muy difícil determinar si los objetivos que motivaron el cambio normativo se han cumplido satisfactoriamente o si la finalidad de la modificación se alcanzará con éxito. Son varios los aspectos que, a corto o a largo plazo, deberán ser analizados. Las prácticas que a raíz del nuevo texto emplee el Tribunal de Justicia, la jurisprudencia que produzca mediante el uso e interpretación de sus normas procesales y los resultados de las estadísticas que anualmente ofrece esta institución se convertirán, a buen seguro, en los principales indicadores.

Obviamente debemos admitir que la reestructuración de la norma adjetiva del Tribunal de Justica supone un avance considerable, al otorgar a las cuestiones prejudiciales la preeminencia que les corresponde. No solamente por tratarse de los procedimientos que en mayor número se plantean ante dicha institución, sino por la relevancia de los mismos en la formación e interpretación del Derecho de la Unión. El Reglamento en vigor reserva un título específico para este tipo de asuntos, al tiempo que hace más completo y explícito su régimen, consolidando las disposiciones de los procedimientos acelerados y de urgencia, que en la norma derogada aparecían como

${ }^{64}$ Se trata del artículo 123 octavo del RPTJd, que no tiene equivalente en el actual. Véase BROBERG y FENGER, Preliminary References..., op. cit., pp. 16-17.

65 El artículo 208 del RPTJ constituye una novedad. Se pretenden esbozar en él los aspectos esenciales del procedimiento a seguirse en el supuesto de que un Estado miembro presentara al Tribunal una petición para que verificase el respecto de las disposiciones de procedimiento establecidas en el artículo 7 del TUE. 
añadidas, e integrando textos normativos dispersos, como los que anteriormente fijaban el contenido mínimo indispensable de toda petición de decisión prejudicial. De forma similar, la regulación del recurso de casación ha sido notablemente mejorada, especialmente en lo referente a la figura de la adhesión.

También debemos valorar positivamente la clarificación respecto de las reglas anteriores, mediante la distinción entre las normas procesales comunes, aplicables a todos los tipos de procedimiento, y las específicas de cada uno de ellos. La asignación de un título y una numeración a cada uno de los artículos, si bien amplía la extensión del Reglamento, facilita mucho más su lectura y localización. Se han eliminado, asimismo, normas en desuso o superfluas; y se ha mejorado, en general, la redacción de los preceptos a favor de los agentes jurídicos y de los justiciables que deben hacer uso del texto. En este sentido, también se ha conseguido armonizar y unificar el empleo de determinados términos jurídicos, adaptándolos a la actualidad. Aun así, no se ha erradicado totalmente la imprecisión de alguna disposición, como la relativa a los criterios para decidir el orden de prioridad de los asuntos o las referentes a las facultades que en determinados trámites se conceden al Presidente del Tribunal o a los Presidentes de Sala, que quedan así excesivamente inconcretas.

En cualquier caso, es prematuro valorar hasta qué punto el nuevo Reglamento podrá satisfacer la pretensión del Tribunal de Justicia de mejorar su capacidad para resolver en un plazo razonable los asuntos que se le someten. En primer lugar, porque algunas de las medidas que al respecto se prevén en el nuevo texto no son sino la consolidación prácticas empleadas con anterioridad a su entrada en vigor y que, por lo tanto, difícilmente van a conllevar cambios de tendencia. Se trata, por ejemplo, de la posibilidad del Tribunal de adoptar decisiones que limiten la longitud de los escritos procesales, o que resuelvan la no celebración de vistas o la facultad de responder mediante auto motivado a cuestiones prejudiciales planteadas por los órganos jurisdiccionales nacionales que no susciten dudas razonables. En segundo lugar, por la ampliación competencial en curso del Tribunal de Justicia.

Sin duda, es esta ampliación competencial de la jurisdicción europea la que planteará una mayor problemática, puesto que operará tanto en el plano cuantitativo como en el cualitativo. En el plano cuantitativo, por la por la avalancha de nuevos Estados miembros de la Unión Europea aún pendiente de cerrar. Y, en el plano cualitativo, por las modificaciones sustantivas y procesales operadas con el Tratado de Lisboa, en concreto:

a) La que faculta al Tribunal de Justicia, con carácter excepcional, para conocer de los recursos de anulación que se interpongan por aquellas personas físicas o jurídicas que se ven afectadas por medidas adoptadas en el ámbito de la PESC (Política Exterior y de Seguri- 
dad Común), cuando las mismas puedan resultar restrictivas de sus derechos, según lo establecido en el artículo 275 del TFUE. No obstante, el Tribunal sigue sin ser competente para pronunciarse en dicho ámbito con carácter general.

b) La que, con la supresión del régimen especial derivado del artículo 35 del TUE, en materia de cooperación judicial y policial en materia penal, y sin perjuicio del régimen transitorio, extiende la competencia del Tribunal de Justicia en dicha materia, con la salvedad de lo dispuesto en el artículo 276 del TFUE. Por lo tanto, el Tribunal de Justicia adquiere competencias para conocer de los recursos por incumplimiento. Además se otorga legitimación activa a los particulares para la interposición de recursos de anulación y se instaura la obligatoriedad de las cuestiones prejudiciales en este ámbito, antes de carácter potestativo.

c) La que permite a todos los órganos jurisdiccionales plantear cuestiones prejudiciales respecto a la política de inmigración, a la política de asilo y a la cooperación judicial en materia civil, que en el antiguo artículo 68 del TCE limitaba a las últimas instancias judiciales.

d) La que concede legitimación activa a toda persona física o jurídica para interponer recursos de anulación contra los actos reglamentarios que la afecten directamente y que no incluyan medidas de ejecución, mediante la introducción del párrafo cuarto del artículo 263 del TFUE, así como la que posibilita a los parlamentos estatales instar a sus Gobiernos a que interpongan un recurso de anulación contra los actos legislativos de la Unión Europea contrarios al principio de subsidiariedad.

e) Y, en último lugar, la que obliga al Tribunal de Justicia a pronunciarse a la mayor brevedad cuando se le plantee una cuestión prejudicial en asuntos pendientes ante un órgano jurisdiccional estatal en relación a una persona privada de libertad, de conformidad con el último párrafo del artículo 267 del TFUE.

Todo indica, pues, que el número de asuntos que en un futuro se presenten ante el Tribunal de Justicia va a ser muy superior a los que dicha institución debía resolver con anterioridad al Tratado de Lisboa. En consecuencia, resulta dudoso que el nuevo Reglamento de Procedimiento sea eficaz para que el juez europeo pueda absorber y gestionar correctamente todos los procedimientos que ahora le competen. Más bien se corre el riesgo de iniciar un nuevo período en el que el Tribunal de Justicia tenga dificultades para dedicarse de manera adecuada a la resolución de controversias, estableciendo el justo equilibrio entre la libertad individual y el interés general de la Unión Europea. 